\title{
The footprint of surface modification treatments on the corrosion-fatigue of AA7075-T651
}

\author{
Nikolaos Michailidis ${ }^{1,2}$, Antonios Ragousis ${ }^{1}$, Fani Stergioudi $^{* 1,2}$, Homero Castaneda ${ }^{3}$ \\ ${ }^{1}$ Physical metallurgy Laboratory, Dept. of Mechanical Engineering, School of Engineering, Aristotle \\ University of Thessaloniki, 54124 Thessaloniki, Greece \\ ${ }^{2}$ Center for Research \& Development on Advanced Materials - CERDAM, 57001 Thermi- \\ Thessaloniki, Greece \\ ${ }^{3}$ National Corrosion and Materials Reliability Center, Texas A\&M University, College Station, TX \\ 77843-3003, USA
}

\begin{abstract}
Different manufacturing and surface modification treatments distinctively affect the surface characteristics and microstructure of the workpiece, having a different impact on their effective life-span. The corrosion-fatigue behavior of as-machined (wire-EDM), blasted and anodized aluminum alloy 7075-T651 was investigated using $3.5 \mathrm{wt} \% \mathrm{NaCl}$ aqueous solution and distilled water as corrosive media. An in-situ corrosion-fatigue device capable of producing cyclic loads in a corrosive solution was employed, coupled with FEM analysis. Blasting process offered a prolongation of the corrosion-fatigue life-span in both corrosive media, when compared to the as-machined samples under identical conditions. Anodizing had a deleterious effect in all the examined cases.
\end{abstract}

\section{Introduction}

The aluminum alloy 7075-T651 is among the most widely used alloys in aerospace applications up to date, due to its light weight and high strength. The AA7075-T651 contains a high number of intermetallic particles that are detrimental to the corrosion properties of the alloy. Consequently, the development of appropriate surface modification treatments that can improve the corrosion resistance and fatigue strength is pivotal for engineering applications $[1,2]$.

Anodizing is a common protection method against corrosion. Additional benefits from anodizing are among others the increased abrasion resistance and paint adhesion, the improvement in adhesive bonding and the ability to provide electrical insulation [3]. Although the anodic aluminum oxide layer adheres well on the substrate layer, due to its compact inner layer which is beneficial to corrosion resistance, the porous outer layer can lead to the propagation of cracks into the substrate, that are easily formed due to its brittle nature. This, results in a dramatic reduction of the fatigue durability of the metal [4].

* Corresponding author: fstergio@auth.gr 
Shot peening or blasting is commonly used to prolong fatigue durability. Shot peening gives also promising results in terms of corrosion fatigue properties for applications where fatigue takes place in a corrosive environment. It has been proven to improve corrosion resistance by reducing the number of pits created on the surface due to localized corrosion [5].

In this research, the effect of different surface modification treatments on the surface characteristics and corrosion fatigue properties of AA 7075-T651 is investigated. More specifically, as-machined (wire-EDM), blasted and anodized samples of AA 7075-T651, were tested to assess their electrochemical stability and corrosion fatigue properties in deionized water and chloride environment (3.5 wt $\% \mathrm{NaCl}$ aqueous solution). An in-situ corrosion-fatigue device capable of producing cyclic loads in a corrosive solution was employed. The experimental process was simulated through finite element method (FEM) to determine the developing stress fields and elucidate crack propagation. The fractured surfaces were studied by scanning electron microscopy (SEM) to investigate crack growth mechanisms.

\section{Materials and methods}

The aluminum alloy 7075-T651 was used as a test material (chemical composition: 1.2$2 \% \mathrm{Cu}, 2.1-2.9 \% \mathrm{Mg}, 0.3 \% \mathrm{Mn} \max , 0.4 \% \mathrm{Si} \max , 0.5 \%$ Fe max, 0.18-0.28\% Cr, 5.1-6.1\% $\mathrm{Zn}, 0.2 \%$ Ti max. 0.5 max, other, Al-balance), having yield stress and ultimate tensile strength of approximately 503 and $572 \mathrm{MPa}$, respectively. C-ring specimens were machined according to the ASTM G38-01 standard, oriented in such a way that the direction of principal stress (parallel to the stressing bolt) is in the direction of minimum resistance to stress-corrosion cracking [6]. The outer diameter, thickness and width of the C-ring were set to $19,1.5$ and $19 \mathrm{~mm}$, respectively.

Type II sulfuric acid anodizing was carried out on some of the C-ring specimens prior to corrosion fatigue testing, using analytical grade reagents. The specimens were degreased in acetone prior to anodizing, then etched in $5 \mathrm{wt} \% \mathrm{NaOH}$ at $55-60^{\circ} \mathrm{C}$ for $120 \mathrm{~s}$ and dismutted in $10 \mathrm{wt} \% \mathrm{HNO}_{3}$ for $120 \mathrm{~s}$. The anodizing bath consisted of $\mathrm{H} 2 \mathrm{SO} 4(15 \% \mathrm{vol})$. The applied constant current density was $1.5 \mathrm{~A} / \mathrm{dm}^{-2}$ while the anodizing time was set to $30 \mathrm{~min}$. Following the anodizing, the samples were sealed in distilled water at $100^{\circ} \mathrm{C}$ for $30 \mathrm{~min}$.

Prior to corrosion fatigue testing, a blasting process was carried out on several C-ring specimens. The medium used for the blasting process was fine glass beads with a nominal diameter of $100 \mu \mathrm{m}$ to avoid iron contamination. The blast media was pneumatically accelerated by compressed air ( 6 bar) and projected by a nozzle onto the specimen (maintaining a constant distance of around $15 \mathrm{~mm}$ ) in a direction vertical to their surface for 5 min.

Corrosion fatigue tests were performed under constant amplitude loading of $250 \mathrm{~N}$ (cyclic load ratio $\mathrm{R}=0$ ), at a frequency of $40 \mathrm{~Hz}$, employing at least 5 specimens for each testing condition. Distilled water and a $3.5 \mathrm{wt} \% \mathrm{NaCl}$ aqueous (distilled water) solution were used as corrosive environments, prepared at $20^{\circ} \mathrm{C}$. Fatigue tests without the presence of any corrosion medium were also performed to C-ring specimens, employing the same loading conditions, so as to distinguish the corrosion effect and its contribution to the final failure of the samples.

The surface of the examined samples was scanned employing the white light confocal microscope $\mu$ SURF (NANOFOCUS AG) to determine the effect of the surface modification treatment on the surface integrity and morphology. SEM observations of the fractured surfaces were also performed for selected samples.

The experimental set-up developed for testing the corrosion fatigue performance of materials is presented in [7]. In-situ electrochemical measurements were performed using a 
Voltalab PGZ 402 advanced electrochemical system. The solutions used during the experiment were aerated and held at $37^{\circ} \mathrm{C}$. The electrochemical experiments were carried out using a conventional three-electrode cell. The reference and auxiliary (counter) electrode were a saturated calomel electrode (SCE) and a platinum wire, respectively. The electrochemical corrosion apparatus was in compliance with the ASTM G 69-97 and ASTM G71-81 standards.

FEM simulations were used to support the experimental process so as to determine the developing stress fields under static loading. The same loading and boundary conditions applied during experimentation were inserted to the FEM model. The C-ring sample was meshed with SOLID186, a higher order 3-D 20-node solid element, whilst an isotropic linear elastic law was used a constitutive law. Young's modulus and Poisson's ratio values for the AA 7075-T651, used for the FEM simulation, were obtained from literature and assigned to $71.7 \mathrm{GPa}$ and 0.33 , respectively.

\section{Results and discussion}

\subsection{Microstructure of the base material and experimental set-up of corrosion fatigue device}

Figure 1 illustrates representative 3D measurements, obtained by the confocal microscopy, to characterize the surface integrity and the topography of the surfaces created via the different surface modification treatments of the AA7075-T651 samples (EDM machining, anodizing and blasting). The wire-EDMed samples present characteristic valleys from the machining process. The blasted sample exhibits a bump surface texture having a double "max valley depth" value compared to the wire-EDMed sample. The anodized sample presents several pits and pores. For the AA 7xxx series, it is documented in literature $[3,8]$ that the long pickling time prior to anodizing results in the formation of pits due to the reaction of intermetallic particles with the pickling agent.

The microstructure of the initial material used is shown in Figure 2. Recrystallized and unrecrystallized grains, elongated in the rolling direction are visible. Spheroid intermetallic precipitates are homogeneously dispersed in the Al matrix. These intermetallic particles are produced during artificial aging and their presence results in the increase of the fatigue strength. This is attributed to their ability of hindering the movement and shifting of dislocations. However, they decrease the corrosion resistance due to their tendency to act as initial sites for dissolution and galvanic corrosion.

A schematic representation of the experimental set-up developed for the in-situ measuring of corrosion fatigue properties of the C-ring samples is illustrated also in Figure 2. The forces are exerted on the transversal direction relative to the microstructural grains, which is the direction of maximum strength for the AA 7075-T651. 


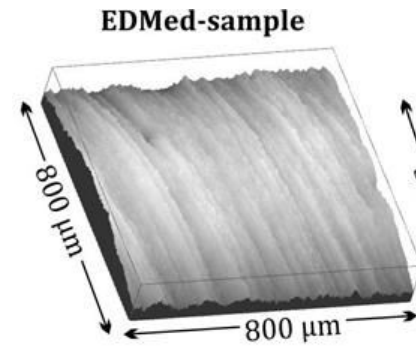

max peak height: $7 \mu \mathrm{m}$ max valley depth: $12 \mu \mathrm{m}$
Blasted sample

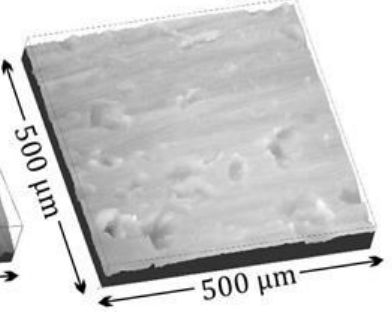

max peak height: $11 \mu \mathrm{m}$ max valley depth: $23 \mu \mathrm{m}$

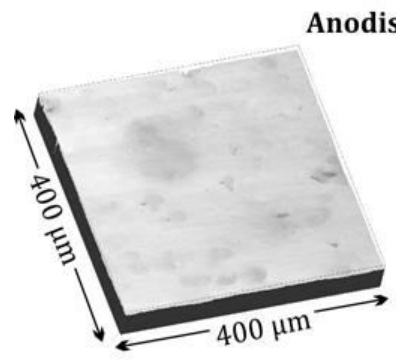

sed sample

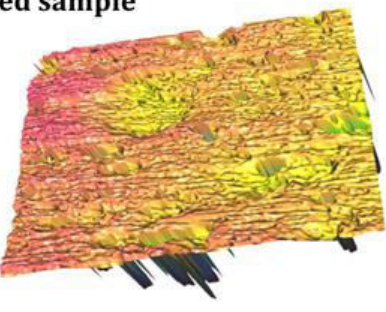

max peak height: $12 \mu \mathrm{m}$ max valley depth: $62 \mu \mathrm{m}$

Fig. 1. Confocal microscopy images of the variously surface-treated samples, prior to corrosion fatigue testing.
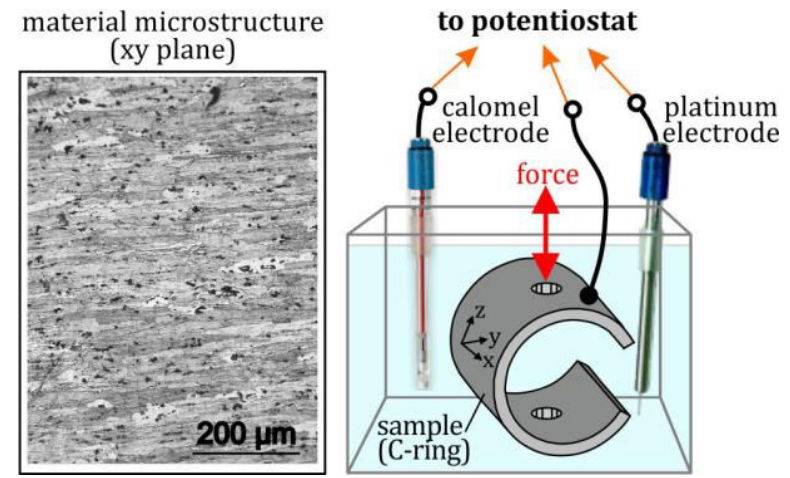

Fig. 2. Microstructure of AA7075-T651 and experimental setup for testing the in-situ corrosion fatigue.

\subsection{FEM simulation results}

Figure 3 shows the equivalent von Mises stress field developed on the AA 7075-T651 C-ring under a peak load of $250 \mathrm{~N}$. The calculated stress field developed is highly inhomogeneous. Throughout the specimen thickness a stress gradient is observed. On its outer surface, maximum tensile stresses are developing, while on the inner one, maximum compressive ones.

Around the circumference of the C-ring stress variations are also visible from a maximum at the middle of the $\mathrm{C}$-ring arc to almost zero at the holes. Variations of the von Mises stresses are also observed on the outer surface over the width of the ring. The von Mises stresses present slightly higher values towards the edges rather than at the middle. This observation advocates that crack initiation does not necessarily propagate 
perpendicularly to the applied load. Nevertheless, this area of the maximum stresses, indicates the area of crack initiation, which was also confirmed by experimental observation of the samples. Due to the elongated grains of the AA 7075-T651 (see figure 2 ), the prediction of the crack evolution becomes even more complicated.

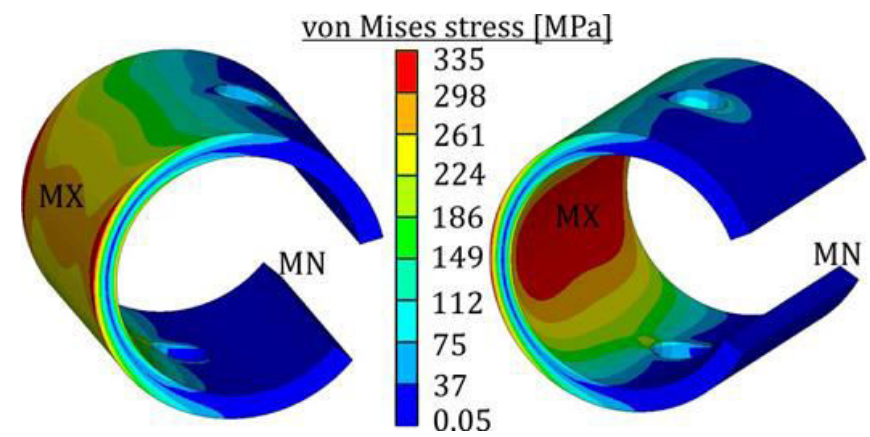

Fig. 3. FEM-determined von Mises stresses developed under a load of $250 \mathrm{~N}$ for the AA-7075-T651 C-ring specimen.

\subsection{Corrosion fatigue behaviour}

Corrosion fatigue tests were designed and performed for a minimum of $3 * 106$ cycles, unless prior failure occurred. All the specimens were tested under the same amplitude loading of $250 \mathrm{~N}$. The corrosion fatigue results, in terms of number of cycles to failure, of the examined treated and untreated C-ring samples are summarized in Figure 4, for two corrosive media (distilled water and $3.5 \mathrm{wt} \% \mathrm{NaCl}$ aqueous solution). To facilitate an ease comparison of the results, fatigue tests under the same loading conditions without the presence of any corrosion medium are also shown. The blasted specimens exhibit improved corrosion fatigue performance, in comparison with the untreated ones, in both corrosive environments. On the contrary, anodizing process has a detrimental effect on corrosion fatigue performance of the AA 7075-T651 samples.

The pronounced deleterious action of the coating layer formed by the anodizing process is denoted by the more than a ten-time decrease of the fatigue life. The reduction in fatigue resistance when employing an anodic coating on aluminum alloys is reported by numerus studies. The brittle and porous nature of the anodizing layer is highly responsible for this fatigue susceptibility [8-12]. Additionally, the anodized layer is susceptible to microcracking. These microcracks are formed on the oxide layer due to the difference in thermal expansion coefficients between the coating layer and the aluminum substrate.

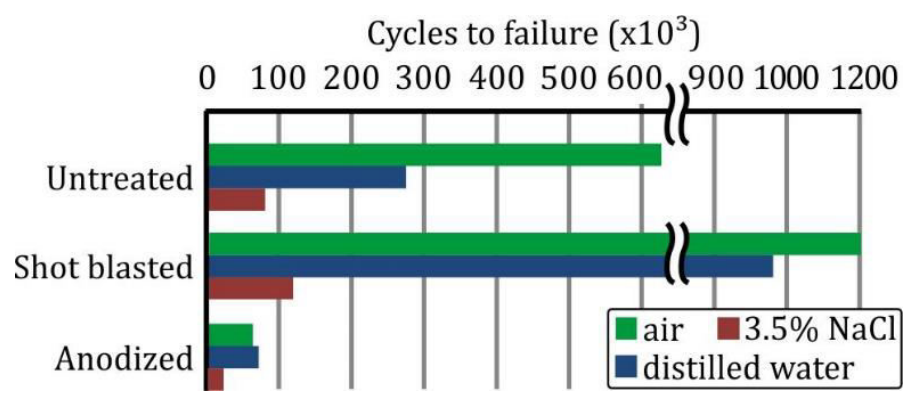

Fig. 4. Average number of cycles to failure in corrosion fatigue testing of AA7075-T651 under different surface treatments and corrosive environments. 
Induced tensile residual stresses on the coating can also aggravate the fatigue strength $[10,12]$. The anodizing coating is highly adhered to the base aluminum alloy which implies that the surface cracks, created during the sealing process, propagate unconstrained into the substrate.

When applying a blasting process, an increase of 3.5 times was documented in the corrosion fatigue life of the AA7075-T651, for the corrosive environment of distilled water (from $2.8 * 10^{5}$ to $9.6^{*} 10^{5}$ cycles to failure). On the contrary, a fourfold decrease was noted (from $2.8 * 10^{5}$ to $0.7 * 10^{5}$ cycles to failure) when applying anodizing.

The same trend is evident for the corrosion fatigue performance at $3.5 \% \mathrm{NaCl}$ aqueous solution. Increased corrosion fatigue life for blasted specimen and deterioration of performance in the case of anodized specimens is documented. The corrosion fatigue life for the anodized specimens was approximately 3.9 times lower, compared to untreated ones. This reduction in corrosion fatigue life is nearly identical to that observed when using distilled water, indicating that there is a negligible dependency of corrosion fatigue life to the corrosive environment for the anodized samples. Under the same corrosive conditions, for the blasted specimens, the average number of cycles to failure was approximately $50 \%$ higher than that of the untreated ones $\left(1.16 * 10^{5}\right.$ from $\left.0.79 * 10^{5}\right)$.

Pits in the anodized sample were verified by confocal microscopy. Those pits act as stress concentration sites, reducing fatigue performance by facilitating crack initiation [4]. During corrosion fatigue, formation of pits is also possible when using the $\mathrm{NaCl}$ solution, due to the different electrochemical potential between the constituent particles and the matrix thus resulting in localized corrosion pits $[13,14]$. However, the duration of the tests was relatively small. Therefore, there was short time for pits to form and negatively affect corrosion fatigue performance.

\subsection{Electrochemical results}

The surface damage and the synergetic effect of the corrosion resistance and fatigue life can be monitored by following the open circuit potential (OCP) fluctuations (in-situ), since OCP is sensitive enough to capture repassivation phenomena that are related to the local failure of the oxide layer.

Figure 5 shows a representative OCP curve as a function of time, obtained during cyclic loading for the AA7075-T651. Prior the dynamic loading, the C-ring specimens were emerged into the corrosive solution for an adequate time, in order for the OCP to reach a steady-state value, which was approximately $-760 \mathrm{mV}$. The initiation of the cyclic loading was accompanied by a subsequent smooth and gradual increase of the OCP. During the whole duration of the corrosion fatigue experiment there was no restoration of the OCP to the initial (prior-to-testing) value. An abrupt OCP shift towards more negative values (marked with arrow), was attributed to a local breakdown of the passive oxide film. After this local failure, the OCP increased up to the initial value, which can be ascribed to the formation of passive film on the freshly formed active surface of the sample. The sample failure was accompanied by a drastic potential drop of the OCP. The AA7075 possesses higher OCP values (increased chemical stability) under continuous cycling conditions. Moreover, the OCP curve is relatively smooth with minor variations. Therefore, it is deduced that the oxide film on the alloy exhibits high resistance to the destructive impact of the applied cyclic loading. 


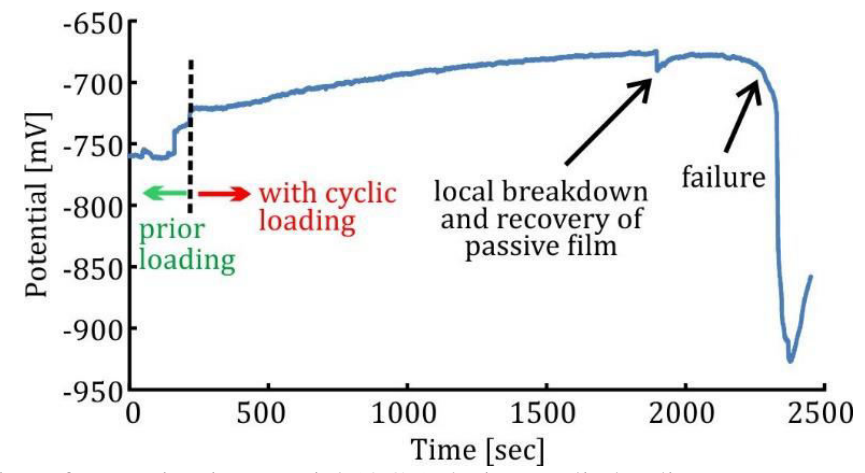

Fig. 5. Evolution of open circuit potential (OCP) during cyclic loading.

\subsection{Fracture analysis}

Figure 6 illustrates the fractured surfaces of all specimens tested at 3.5\% $\mathrm{NaCl}$ aqueous solution. At regions located close to the outer surface of the C-ring, transgranular cracking throughout the length of the specimen (see Figure 6) seems to be prevalent for the untreated and blasted samples. Nevertheless, at regions located at the mid-thickness of the specimen, several differences appear at the fracture surfaces, as presented

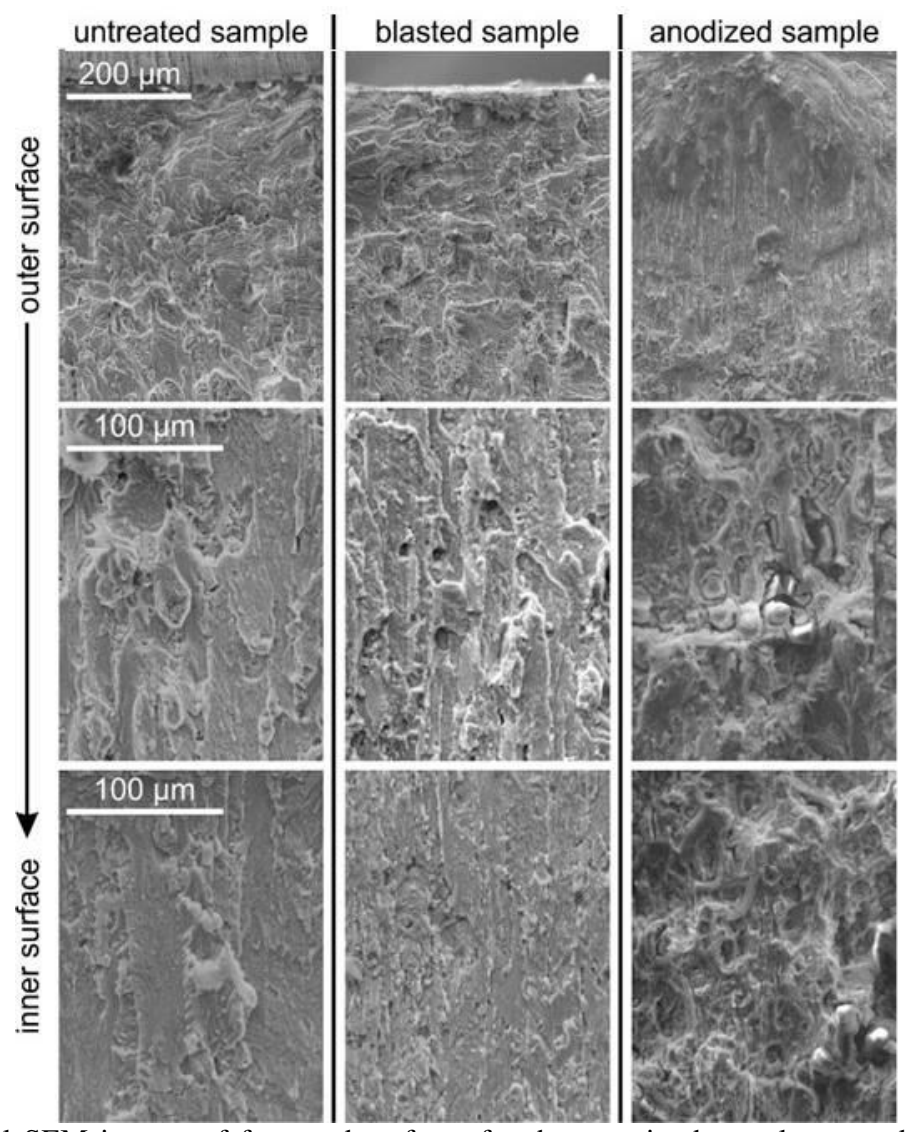

Fig. 6. Typical SEM images of fractured surfaces for the examined samples, tested at $3.5 \% \mathrm{NaCl}$ aqueous solution. 
The specimens seem to experience a more ductile fracture mode close to the outer surface, as indicated by the irregularities of the fractured surface. This may be attributed to the low hydrogen concentration at crack tip, leading thus to minor embrittlement. As the crack propagates through the length of the specimen, a transition to brittle transgranular fracture is observed with intense corrosion pits between the grains. Close to the inner surface, flat cleavage facets are observed which advocates the conclusion that the fracture was mainly transgranular. This result agrees with prior studies reporting that corrosion fatigue fracture of aluminum alloys 7075 is predominantly transgranular $[15,16]$.

SEM images of the anodized fractured surfaces indicate the existence of three distinct facture morphologies throughout the thickness of the C-ring sample. A nucleation site is evident, featuring large river like marks pointing to the initiation site of crack propagation. The participation of the coating in the fracture process is ascertained by the SEM images, since it was observed that the crack originates at the surface. Cavities close to the outer surface are ascribed to corrosion pits and detachment of intermetallic compound. These cavities can act as stress concentration sites, facilitating fatigue crack initiation and promoting crack growth. The second area, appears to be more ductile whilst it features a dimple-containing fractured surface. A transition to brittle transgranular mode is observed in the last region.

\section{Conclusions}

The effect of various surface modification treatments (wire-EDM, blasting and anodizing) on the corrosion-fatigue behavior of aluminum alloy 7075-T651 was investigated using $3.5 \mathrm{wt} \% \mathrm{NaCl}$ aqueous solution and distilled water as corrosive media. The examined blasting process allowed for a prolongation of the corrosion fatigue life of the tested aluminum alloy, at both corrosive environments. On the contrary, anodizing had a detrimental effect on corrosion fatigue life. The macro- and micro- structural fracture analysis indicate similar cracking mechanisms for the untreated and blasted specimens, whereas anodized specimens present different cracking modes.

\section{References}

1. V. Schulze, F. Bleicher, P. Groche, et al., 2016, CIRP Ann.-Manuf. Techn 65, 809 (2016).

2. H. Ohmori, K. Katahira, J. Nagata, et al., CIRP Ann.-Manuf. Techn 51, 491 (2002).

3. M. Shahzad, M. Chaussumier, R. Chieragatti et al., Procedia Engineering 2, 1015 (2010).

4. M. Shahzad, M. Chaussumier, R. Chieragatti et al., Surf. Coat. Tech. 206, 2733 (2011).

5. U. Zupanc, J. Grum, Mater. Process. Tech. 210, 1197, (2010).

6. ASTM G38-01, Standard practice for making and using C-ring stress-corrosion test specimens. ASTM International, USA, (2013).

7. N. Michailidis, F. Stergioudi, G. Maliaris, A. Tsouknidas, Surf. Coat. Tech. 259, 456 (2014).

8. S.A. Khan, Y. Miyashita, Y. Mutoh, T. Koike, 2008, Mat. Scie. Eng. A 498, 377 (2008).

9. J.A.M. de Camargo, H.J. Cornelis, V.M.O.H. Cioffi, M.Y.P. Costa, Surf. Coat. Tech. 201, 9448 (2007).

10. E. Cirik, K. Genel Surf. Coat. Tech. 202, 5190 (2008).

11. E. Lee, Y. Jeong, S. Kim, Metall Mater Trans A 43, 2001 (2012). 